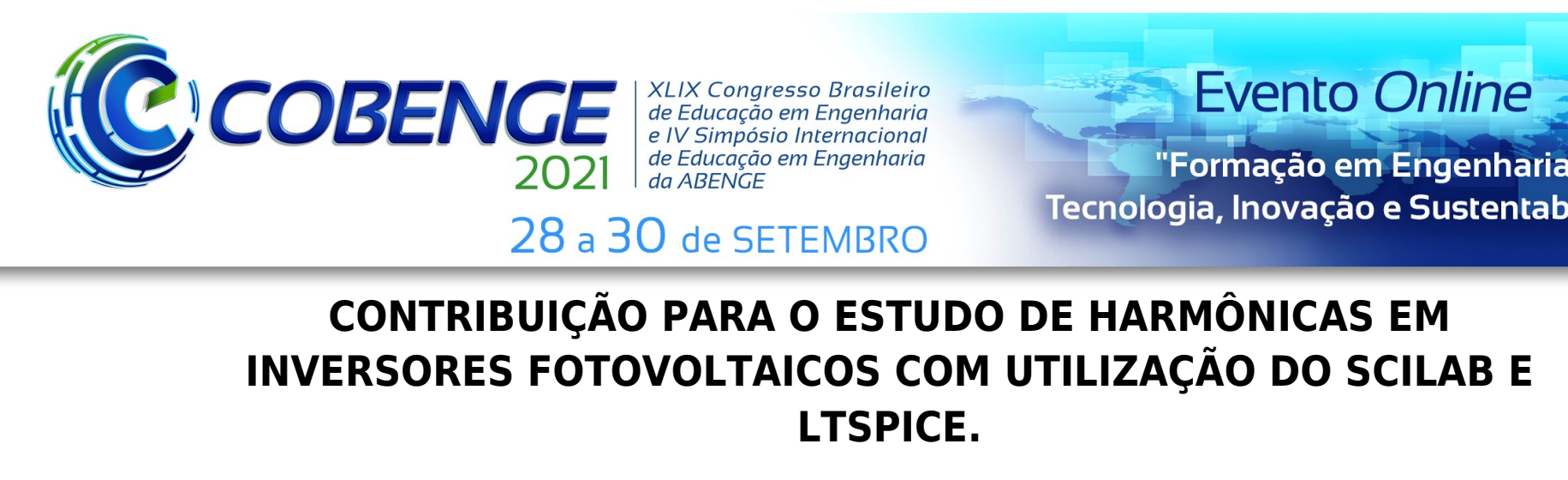

DOI: 10.37702/2175-957X.COBENGE.2021.3442

Bruno de Souza Marques - smarques.bruno@gmail.com

Universidade Cidade de São Paulo UNICID

Rua Antônio Cinelli 17

03677-180 - São Paulo - SP

Carlos Eduardo Freitas - carlos.freitas@unicid.edu.br

Universidade Cidade de São Paulo UNICID

Av Leonor 33

07025-200 - Guarulhos - SP

Rodrigo Amorim Juvencio - raj_roots@hotmail.com

Universidade Cidade de São Paulo UNICID

Rua São Francisco 101

08745-250 - Mogi das Cruzes - SP

Vinicius Moura Ferreira - vinicius_femoura@hotmail.com

Universidade Cidade de São Paulo UNICID

RUA DELI 213

03563-320 - SAO PAULO - SP

Resumo: O modelamento de sistemas elétricos para geração de energia engloba diversos métodos, desde a utilização de softwares computacionais até análises laboratoriais para que se garanta a qualidade na geração. Conhecer mais a fundo, através de argumentos técnicos e científicos, os sistemas de geração fotovoltaica, é de fundamental importância uma vez este meio de fornecimento alternativo de energia altera o padrão estrutural da rede pública e privada do sistema elétrico de potência. Com o intuito de poder mensurar o impacto destas alterações, é proposto a utilização de duas ferramentas gratuitas, o Scilab em sua versão 6.1.0 e o software de circuitos elétricos LTSpice em sua versão XVII, onde se pode simular tipos de modulações existentes nos conversores inversores de tensão CCCA, harmônicas geradas de acordo com cada tipo de modulação e os cálculos 
referentes aos circuitos apresentados. Tais parâmetros são de fundamental importância quanto a qualidade da energia elétrica.

Palavras-chave: Harmônicas. Inversores. Fotovoltaicos. Modulações. PWM. 


\section{CONTRIBUIÇÃO PARA O ESTUDO DE HARMÔNICAS EM INVERSORES FOTOVOLTAICOS COM UTILIZAÇÃO DO SCILAB E LTSPICE.}

\section{INTRODUÇÃO}

Como iniciativa de tornar cada vez mais acessível as ferramentas de análise, pesquisa e desenvolvimento de projetos de engenharia, foram empregados neste estudo softwares de utilização gratuita, que oportunizam e viabilizam a análise de temáticas diversas no meio acadêmico.

A demanda pelo controle de energia elétrica para sistemas de acionamentos de máquinas elétricas e controles industriais existe há muitos anos e isto conduziu ao desenvolvimento do antigo sistema Ward-Leonard para se obter uma tensão CC variável utilizada no controle de máquinas CC. A eletrônica de potência revolucionou o conceito de controle de potência para a conversão de energia e para o controle de máquinas elétricas (RASHID, 1999).

Através de uma análise básica, percebe-se que grandíssima parte da eletrônica de potência é fundamentalmente ligada ao acionamento dos dispositivos semicondutores. Portanto, para que estes dispositivos possam fornecer uma capacidade cada vez maior de chaveamento, a tecnologia deve contribuir quanto às condições nominais de funcionamento, velocidade de trabalho, entre outros, e é o que vem acontecendo.

\section{MODULAÇÕES DE CONVERSORES CC-CA DE TENSÃO MONOFÁSICO}

Atualmente muitos circuitos são alimentados com tensão senoidal da rede pública de distribuição e também por conversores estáticos CC-CA para controlar a tensão na carga e demais grandezas. Normalmente para efetuar o controle destas grandezas nos circuitos inversores, são implementadas técnicas de modulações PWM, controle por defasagem entre outros. O conteúdo harmônico, de acordo com a modulação escolhida, representa exatamente o quanto o sinal de tensão ou corrente é afetado em decorrência da técnica de modulação utilizada. O programa utilizado para elaborar os circuitos e análises computacionais foi o software LTSpice versão XVII e para as modelagens matemáticas foi utilizado o Scilab versão 6.1.0.

\subsection{Controle da Tensão por Defasagem}

Este tipo de método é executado por meio de dois sinais de onda quadrada defasados de um ângulo $\theta$ (theta), ambos com duty cicle (ciclo de trabalho) de $50 \%$. $O$ deslocamento entre as fases destes sinais podem ser de 0 a $\pi$ (zero à pi), onde através deste deslocamento é modulado o sinal de saída com um ângulo y (gama) de duração do pulso, que também varia de 0 a $\pi$, os pulsos destes sinais $V(v a), V(v b)$ são combinados através de um circuito de controle para acionar as chaves semicondutoras de um driver de ponte completa. Para deslocar o sinal é utilizado o circuito deslocador de fase MFB (Multiple Feed-Back) ou Filtro Passa Todas. 
Figura 1 - Sinais $\mathrm{V}(\mathrm{va}), \mathrm{V}(\mathrm{vb})$ defasados de ângulo $\theta$ de $90^{\circ}$ (LTSpice).

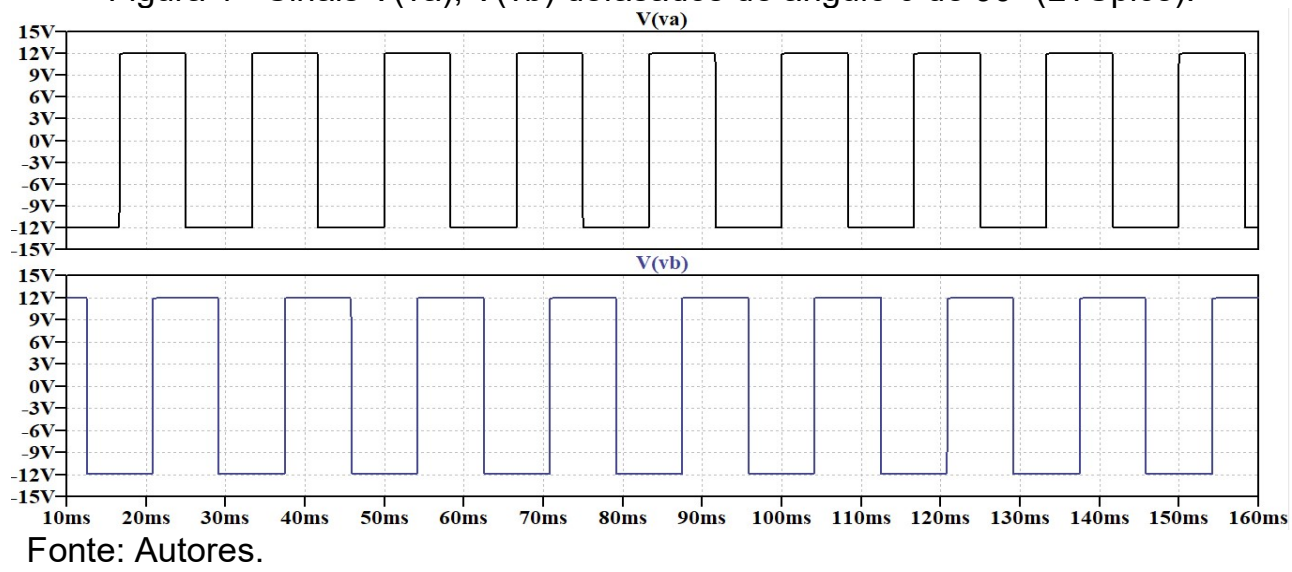

Abaixo, o circuito eletrônico proposto e as formas de onda obtidas da modulação controle da tensão por defasagem.

Figura 2 - Circuito de Modulação Controle da Tensão por Defasagem (LTSpice).

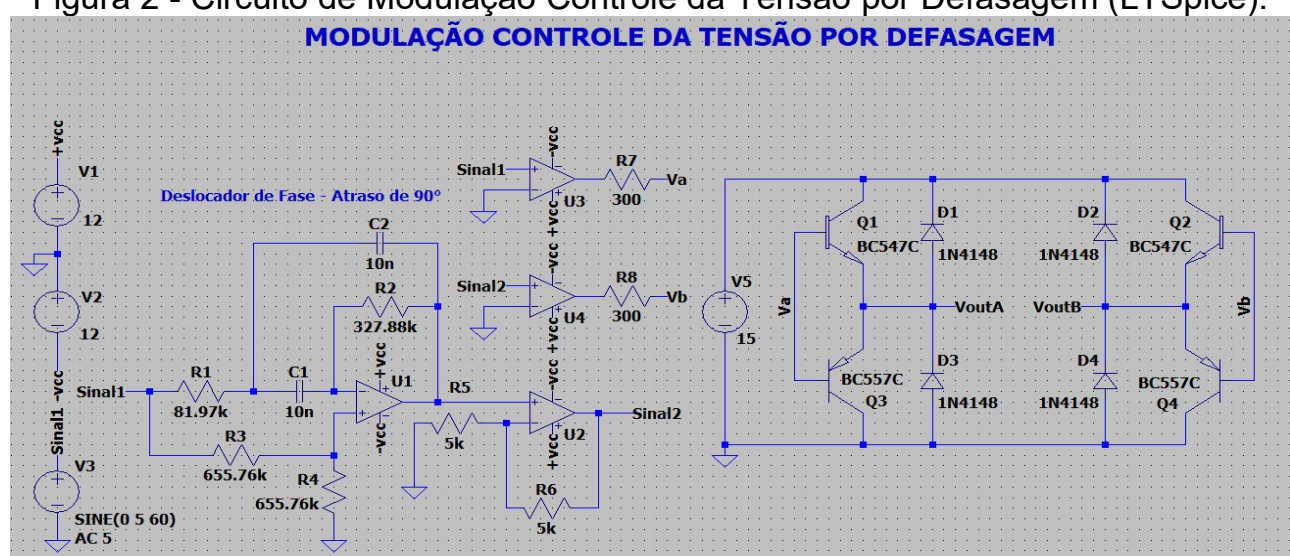

Fonte: Autores.

Neste circuito, uma onda senoidal de referência de $60 \mathrm{Hertz} \operatorname{com} \theta=0$ atuando como entrada do circuito defasador de fase (Phase-Shifting), e em sua saída é entregue o sinal senoidal atenuado com ganho $\mathrm{K}=0,5$ e fase $90^{\circ}$ de atraso com relação a sua entrada, conforme demonstra a figura 3 .

Figura 3 - Formas de Ondas da Entrada/Saída Circuito Phase-Shifting (LTSpice).

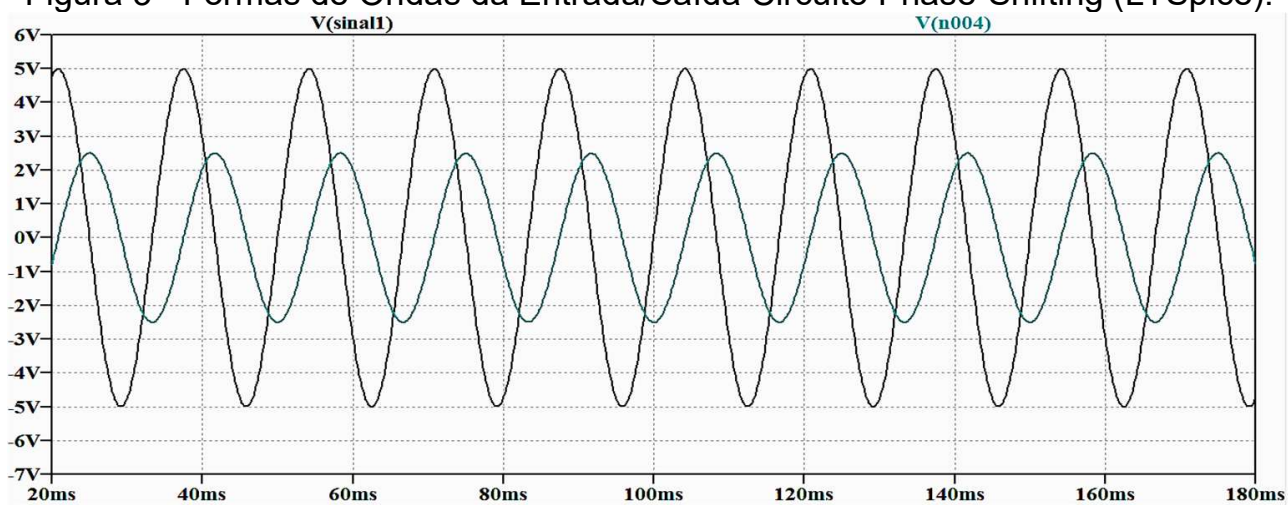

Fonte: Autores.

Curva de módulo e fase são obtidas em função da frequência. Abaixo o gráfico de Bode para demonstrar a saída do sinal atrasado de $90^{\circ}$. 
Figura 4 - Diagrama de Bode do sinal defasado (LTSpice).

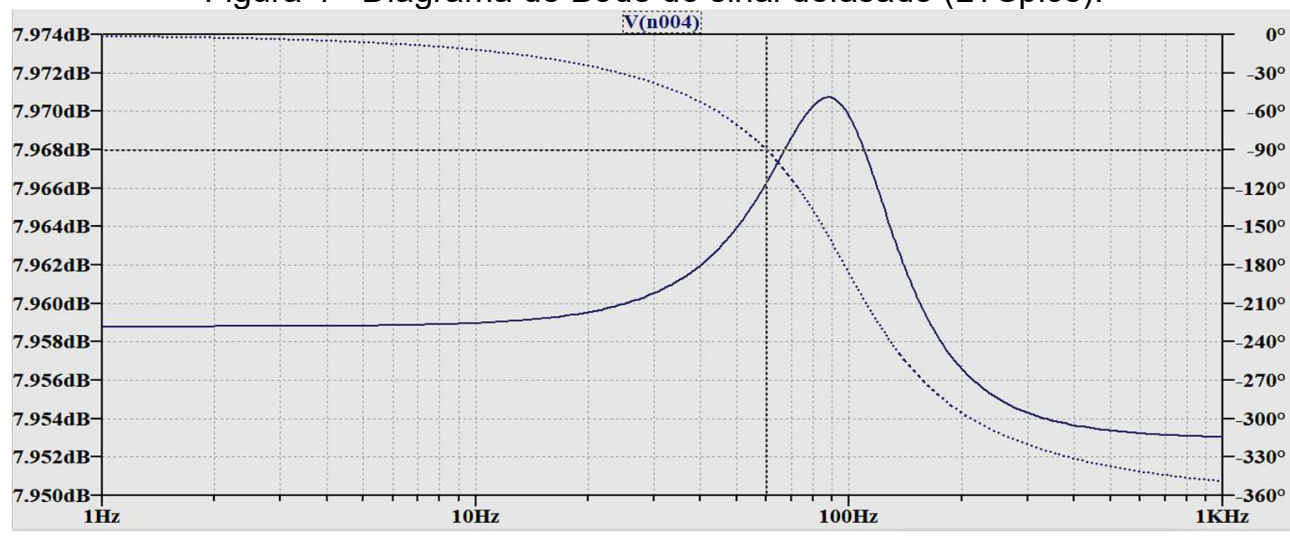

Fonte: Autores.

As formas de ondas abaixo demonstram o funcionamento do circuito montado, tensão na carga com amplitude (+E) e (-E) e ângulo y de $\pi / 2$.

Figura 5 - Sinal de Saída na Carga (LTSpice).

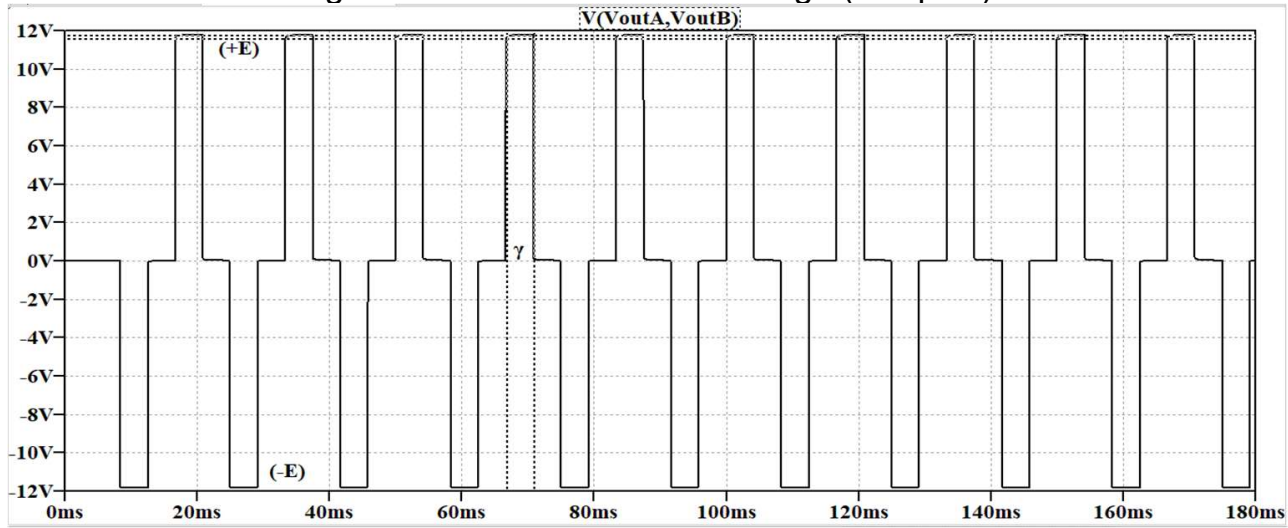

Fonte: Autores.

Conforme (DENIZAR; BARBI, 2011) a tensão de saída na carga é obtida através da série de Fourier:

$$
V(\text { out } A, \text { out } B)=\frac{4 E}{\pi} \sum_{n}^{\infty} \frac{1}{n} \operatorname{sen} \frac{n \gamma}{2} \operatorname{sen}(n \omega t) \quad \text { onde } n=2 K+1 ; K=0,1,2,3 \ldots ., \infty
$$

THD - Modulação Controle da Tensão por Defasagem

A taxa de distorção harmônica total (THD) da tensão de saída desta modulação é dada pela "Equação (2)" e de corrente pela "Equação (3)":

$$
\begin{gathered}
T H D_{V o}=\frac{\sqrt{2}}{4 \operatorname{sen}\left(\frac{\gamma}{2}\right)} *\left[\pi \gamma-8\left(\operatorname{sen}\left(\frac{\gamma}{2}\right)\right)^{2}\right]^{\frac{1}{2}} \\
T H D_{i o}=\left(\frac{1}{I o_{1 e f}}\right) *\left(I o_{e f}^{2}-I o_{1 e f}^{2}\right)^{\frac{1}{2}}
\end{gathered}
$$

$I o_{e f}=$ Corrente eficaz total 
$I o_{1 e f}=$ Corrente eficaz da componente fundamental

\subsection{Modulação PWM Senoidal Dois Níveis}

A Modulação PWM Senoidal é uma técnica de modulação que reduz a valores interessantes as componentes harmônicas de baixa ordem e o fator de distorção. Quanto maior o número de pulsos por período maior será a diminuição das amplitudes dos harmônicos de baixa ordem (FLORERO, 1989).

Segundo (DENIZAR; BARBI, 2011), este tipo de modulação, a tensão instantânea de saída só pode atingir dois níveis, +E e -E.

Podemos definir então com as seguintes inequações:

$V($ out $A$, out $B)=+E$ quando Vsen $>$ VTriang

$V($ out $A$, out $B)=-E$ quando Vsen $<$ VTriang

Figura 6 - Estrutura das ondas da modulação PWM a dois níveis (LTSpice).

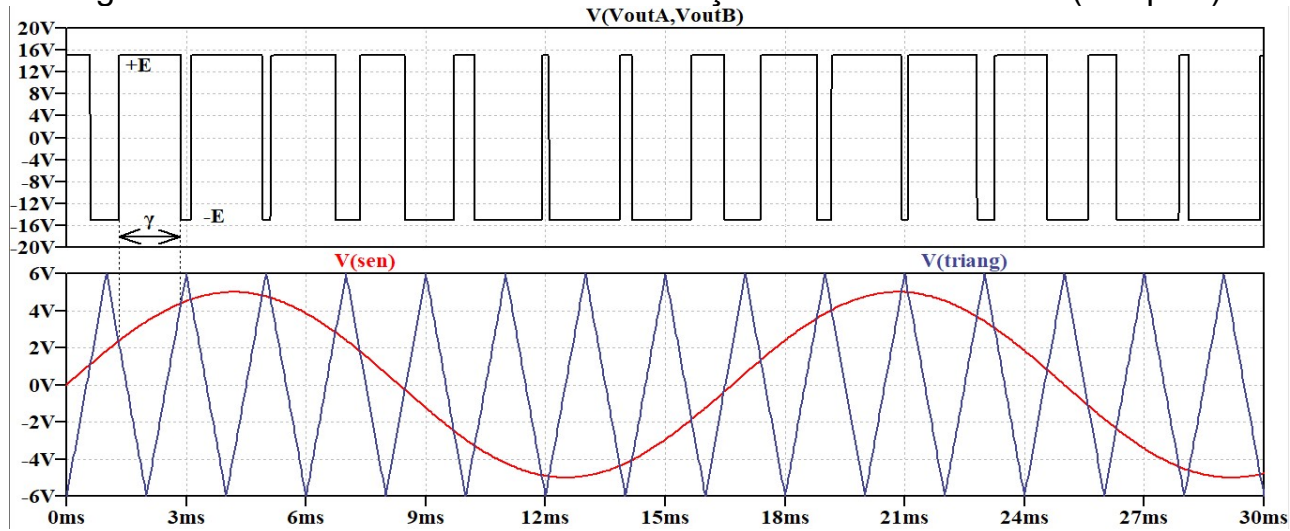

Fonte: Autores.

O PWM1 comanda as Chaves Q1 (NPN) e Q3(PNP), as chaves Q2 (NPN) e Q4 (PNP) são comandadas pelo PWM2. Com o aumento da frequência da onda portadora, aumenta-se o número de pulsos da tensão de saída. Como o aumento dos pulsos está ligado ao teor harmônico da tensão $V$ (outA, outB), quanto maior o número de pulsos, as harmônicas tendem a ir para uma faixa de frequência mais elevada, ficando mais fácil de obter uma senóide pura na saída com filtros.

Figura 71 - Circuito modulação PWM senoidal dois níveis (LTSpice) MODULAÇÃO PWM SENOIDAL A DOIS NÍVEIS.

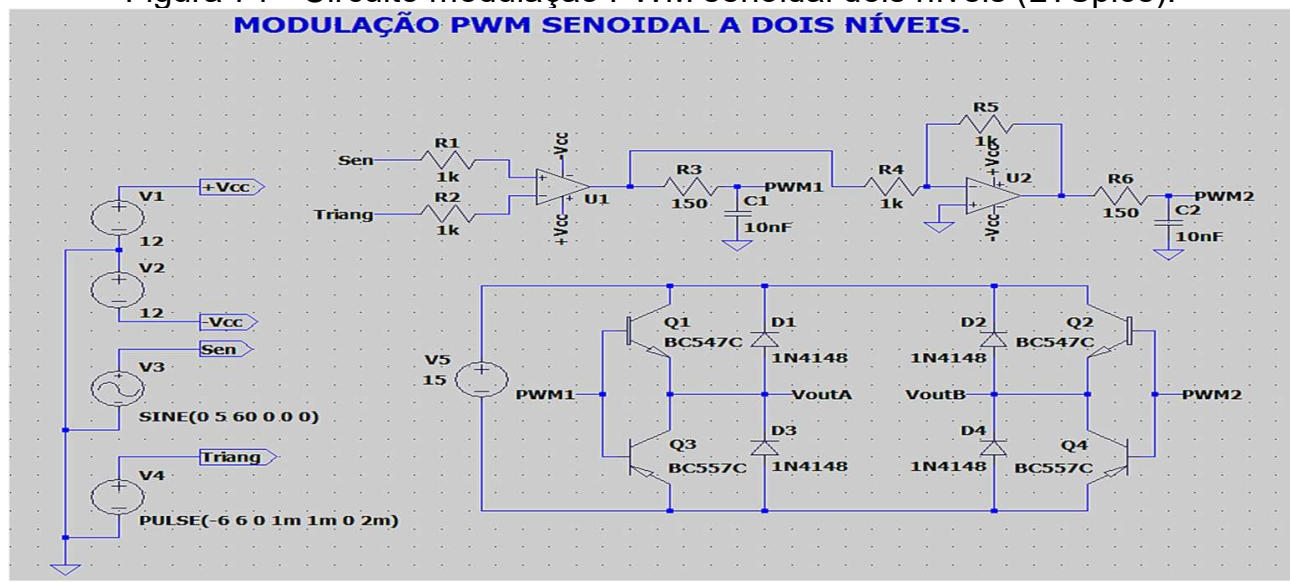

Fonte: Autores. 

de Fourier:

Conforme (DENIZAR; BARBI, 2011), a tensão instantânea da saída é dada pela série

$$
\operatorname{vout}, \operatorname{outB}_{(t)}=\sum_{n}^{\infty} \operatorname{Vout} A, \text { outB }_{\text {nmax }} * \operatorname{sen}(n \omega t) \quad n=2 k+1, \text { sendo } k=0,1,2,3 \ldots \infty
$$

\subsection{Modulação PWM Senoidal Três Níveis}

O circuito SPWM três níveis é semelhante ao dois níveis, difere que o sinal de referência senoidal (Sen) e o triangular (Triang) são comparados em dois circuitos, sendo no segundo com a portadora triangular defasada de $180^{\circ}$.

Figura 8 - Circuito Modulação Senoidal três níveis (LTSpice).

$$
\text { MODULAÇÃO PWM SENOIDAL A TRÊS NÍVEIS. }
$$

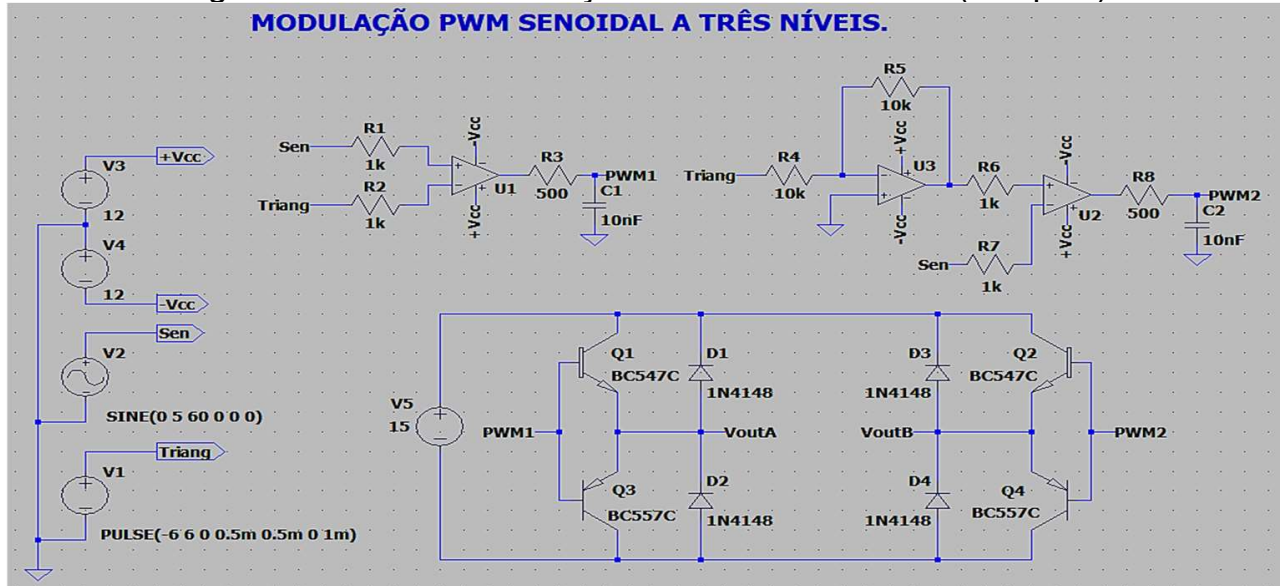

Fonte: Autores.

O sinal do PWM1 é obtido a partir da onda senoidal e da portadora triangular, este comanda as chaves Q1 (NPN) e Q3(PNP). As chaves Q2 (NPN) e Q4 (PNP) são comandadas pelo PWM2 que é obtido através da portadora triangular defasada de $180^{\circ} \mathrm{e}$ da onda senoidal. Chamamos de modulação senoidal três níveis porque a tensão de saída assume +E, 0 e -E, ou seja, três níveis diferentes de tensão.

Figura 9 - Geração dos Pulsos das Chaves (LTSpice).

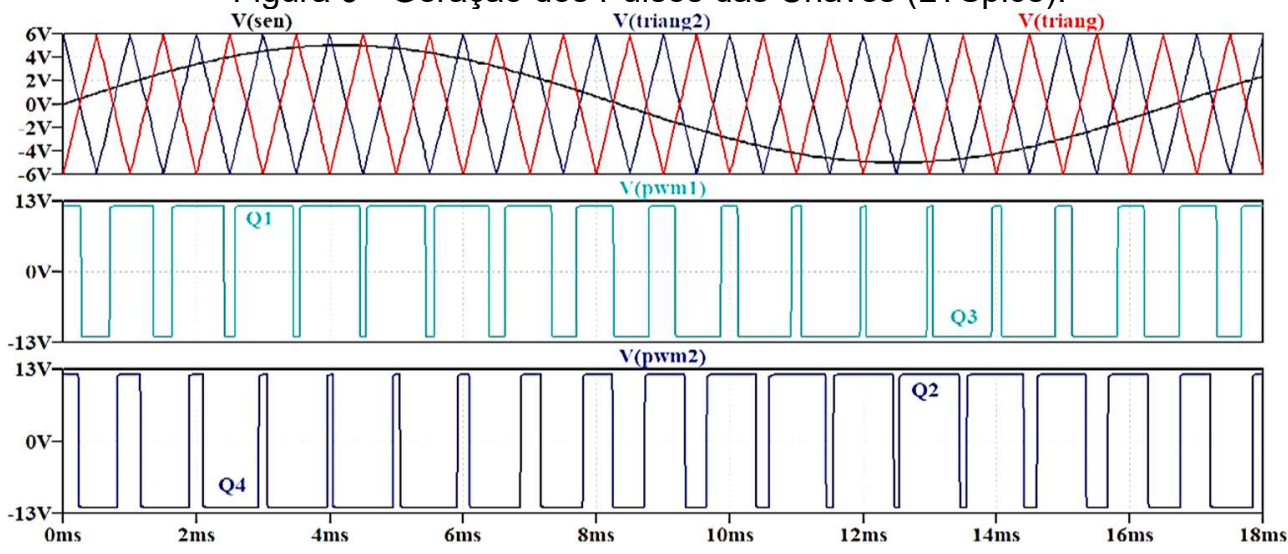

Fonte: Autores.

As formas de onda $V$ (outA,outB), são obtidas obedecendo as seguintes lógicas de controle: 
Se Vsen > Vtriang, então Q1 em condução $\rightarrow V_{p w m 1}$ Se Vsen <Vtriang, então Q3 em condução $\rightarrow V_{p w m 1}$ Se Vsen < Vtriang2, então Q2 em condução $\rightarrow V_{p w m 2}$ $V($ out $A$, out $B)=V(p w m 1)-V(p w m 2)$

Se Vsen > Vtriang2, então Q4 em condução $\rightarrow V_{p w m 2}$

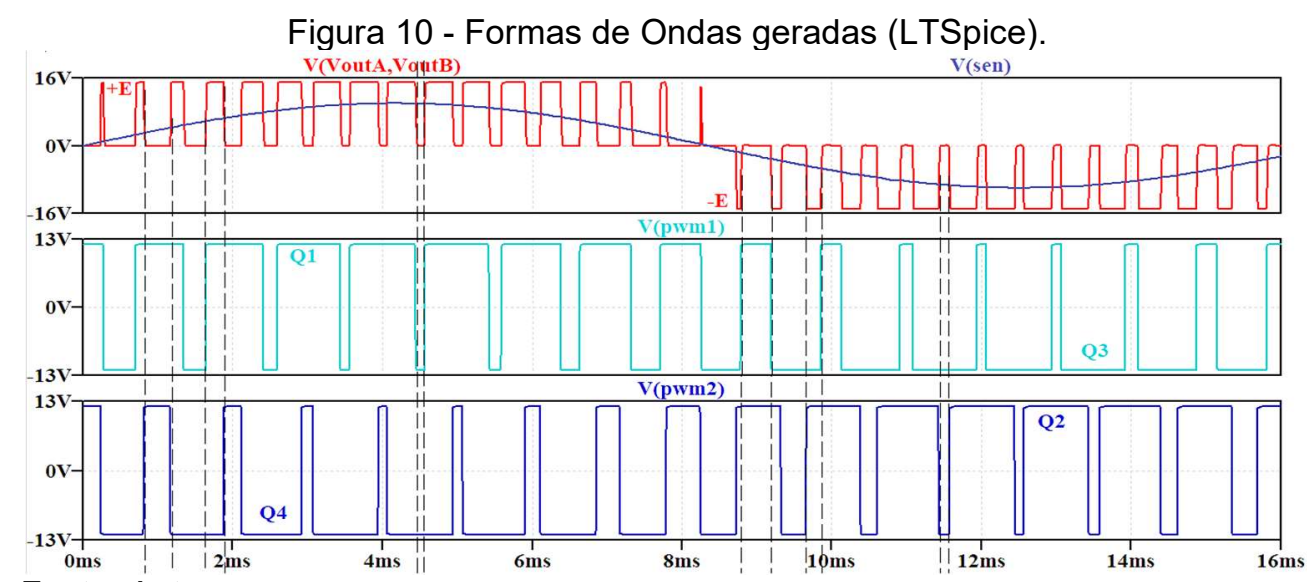

Fonte: Autores.

Durante o semiciclo positivo as chaves Q2 e Q3 estão em modo bloqueio.

$V_{p w m 1}=+; V_{p w m 2}=-\rightarrow$ Vout $A$, outB $=E \rightarrow Q 1, Q 4$ em condução.

$V_{\text {pwm1 }}=+; V_{\text {pwm }}=+\rightarrow$ Vout $A$, outB $=0 \rightarrow$ Q4 bloqueado e Q1, D2 em condução.

$V_{p w m 1}=-; V_{p w}=-\rightarrow$ Vout $A$, outB $=0 \rightarrow Q 1$ bloqueado e Q4,D3 em condução.

Durante o semiciclo negativo as chaves Q1 e Q4 estão em modo bloqueio.

$V_{p w}=-; V_{p w m 2}=+\rightarrow$ Vout $A$, outB $=-E \rightarrow Q 2, Q 3$ em condução.

$V_{\text {pwm } 1}=-; V_{\text {pwm2 }}=-\rightarrow$ Vout $A$, outB $=0 \rightarrow$ Q2 bloqueado e Q3, D4 em condução.

$V_{\text {pwm1 }}=+; V_{\text {pwm2 }}=-\rightarrow$ Vout $A$, outB $=0 \rightarrow$ Q3 bloqueado e Q2, D1 em condução.

Conforme (DENIZAR; BARBI, 2011), a tensão instantânea de saída é dada pela "Equação (4)" expressa em série de Fourier na modulação SPWM três níveis.

Para obter o valor da amplitude da tensão do sinal de saída deve-se aplicar o coeficiente da série de Fourier, considerando cada pulso do semiperíodo que varia de acordo com uma função senoidal, conforme (RASHID, 1999).

$$
V(\text { out } A, \text { outB })_{\text {max }}=\sum_{m=1}^{N} \frac{2 E}{n \pi} * \operatorname{sen}\left(\frac{n \gamma_{m}}{2}\right) *\left[\operatorname{sen} n\left(\alpha_{m}+\frac{\gamma_{m}}{2}\right)-\operatorname{sen} n\left(\alpha_{m}+\pi+\frac{\gamma_{m}}{2}\right)\right]
$$

үm = Largura do m-ésimo pulso da tensão de saída em rad;

am = Tempo de início do pulso ym em rad;

$\mathrm{N}=$ número de pulsos por semiperíodo.

Abaixo temos a tensão eficaz de saída conforme (DENIZAR; BARBI, 2011). 


$$
\text { Vout }, \text { out } B_{\text {ef }}=E * \sqrt{\sum_{m=1}^{N} \frac{\gamma_{m}}{\pi}}=\frac{V(\text { out } A, \text { out } B)_{\text {nmax }}}{\sqrt{2}}
$$

THD - Modulação PWM Senoidal a Dois e Três Níveis

Para estabelecer o THDvo e o THDio, tem-se as equações abaixo:

$$
\begin{aligned}
& \text { THDvo }=\frac{\sqrt{\sum_{n=m f}^{\infty} \text { Vonef }^{2}}}{\text { Vo1ef }} \\
& \text { THDio }=\frac{\sqrt{\sum_{n=m f}^{\infty} \text { Ionef }^{2}}}{\text { Io1ef }}
\end{aligned}
$$

A razão da frequência da modulação é definida pela "Equação 9" sendo $m f \geq 10$ que corresponde a maioria dos conversores CC-CA, conforme (DENIZAR; BARBI, 2011):

$f p$ é a frequência da portadora.

$f$ é a frequência da moduladora.

$$
m f=\frac{f p}{f}
$$

\section{RESULTADOS}

\subsection{Modulação Controle da Tensão por Defasagem}

Figura 11 - Espectro de Frequência (FFT) do sinal com defasagem de $\theta=90^{\circ}$ (LTSpice).

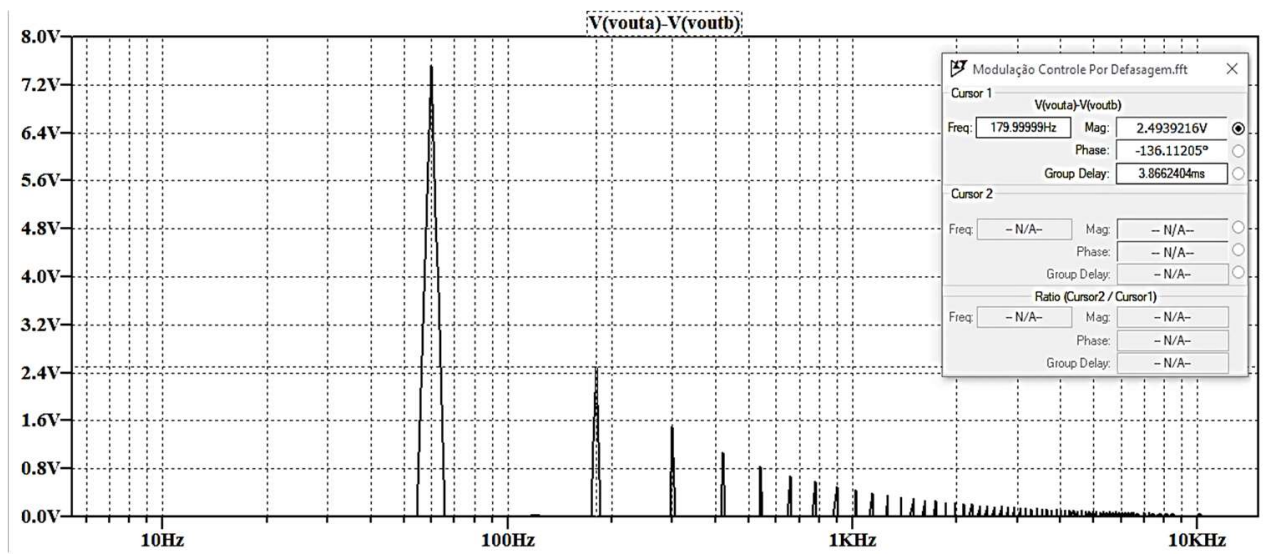

Fonte: Autores.

Tabela 1 - Valores medidos das componentes ímpares até a $19^{\circ}$ (Tensão Eficaz).

\begin{tabular}{|c|c|c|c|}
\hline Componentes $\mathbf{n}$ & $\mathbf{V}\left(\right.$ outA,outB) $\mathbf{n}_{\text {ef }}$ (V) & Componentes $\mathbf{~}$ & $\mathbf{V}\left(\right.$ outA,outB) $\mathbf{n}_{\text {ef }}$ (V) \\
\hline Fundamental & 7,5 & $11^{\mathrm{a}}$ & $675,16 \times 10^{-3}$ \\
\hline $3^{\mathrm{a}}$ & 2,5 & $13^{\mathrm{a}}$ & $580,8 \times 10^{-3}$ \\
\hline $5^{\mathrm{a}}$ & 1,5 & $15^{\mathrm{a}}$ & $493,43 \times 10^{-3}$ \\
\hline
\end{tabular}


28 a 30 de SETEMBRO

Evento Online

"Formação em Engenharia:

Tecnologia, Inovação e Sustentabilidade"

\begin{tabular}{|c|c|c|c|}
\hline $7^{\mathrm{a}}$ & 1,064 & $17^{\mathrm{a}}$ & $445,15 \times 10^{-3}$ \\
\hline $9^{\mathrm{a}}$ & $837,15 \times 10^{-3}$ & $19^{\mathrm{a}}$ & $388,27 \times 10^{-3}$ \\
\hline
\end{tabular}

Fonte: Autores.

\subsection{Modulação PWM Senoidal a Dois Níveis}

Figura 12 - Espectro de Frequência (FFT) modulação SPWM dois níveis (LTSpice).

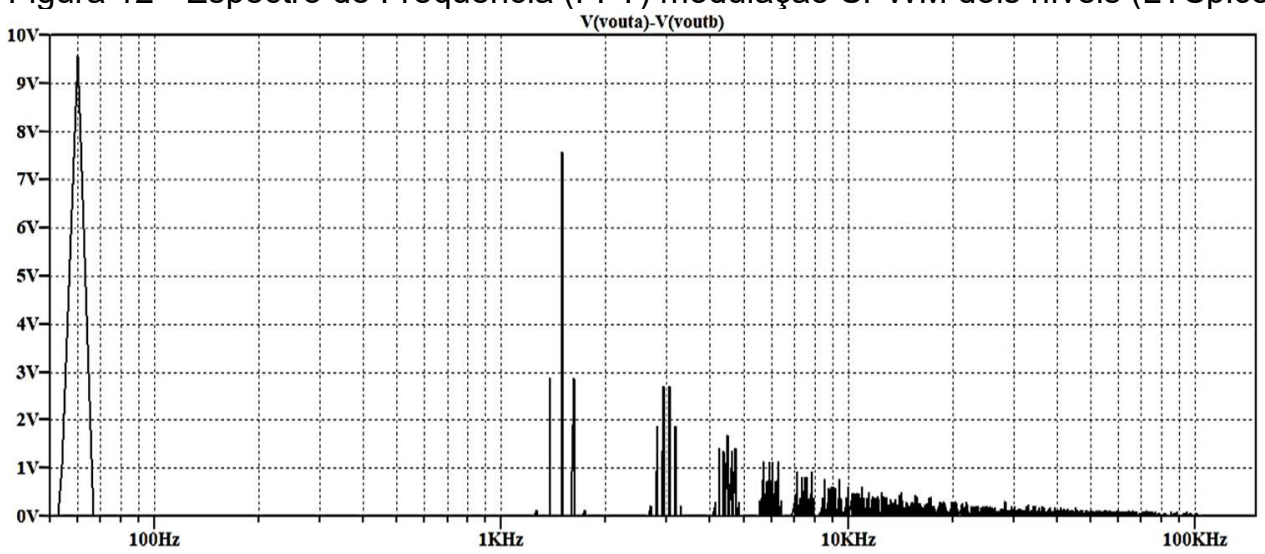

Fonte: Autores.

Tabela 2 - Valores medidos das componentes (Tensão Eficaz)

\begin{tabular}{|c|c|c|c|c|c|}
\hline Componentes & V(outA,outB)n & Frequência & Componentes & V(outA,outB) $\mathbf{n}_{\text {ef }}$ & Frequência \\
\hline Fundamental & 9,57 & 60 & $45^{\text {a }}$ & $226,42 \times 10^{-3}$ & 2700 \\
\hline $21^{\mathrm{a}}$ & $127,32 \times 10^{-3}$ & 1260 & $47^{\mathrm{a}}$ & 1,88 & 2820 \\
\hline $23^{\mathrm{a}}$ & 2,85 & 1380 & $49^{\mathrm{a}}$ & 2,71 & 2940 \\
\hline $25^{\mathrm{a}}$ & 7,57 & 1500 & $51^{\mathrm{a}}$ & 2,71 & 3060 \\
\hline $27^{\mathrm{a}}$ & 2,85 & 1620 & $53^{\mathrm{a}}$ & 1,88 & 3180 \\
\hline $29^{\mathrm{a}}$ & $127,27 \times 10^{-3}$ & 1740 & $55^{\mathrm{a}}$ & $226,35 \times 10^{-3}$ & 3300 \\
\hline
\end{tabular}

Fonte: Autores.

As primeiras harmônicas surgem a partir da $21^{\circ}$ facilitando a implementação do filtro. Quanto maior a frequência de corte, menores são os componentes passivos.

Figura 13 - THD modulação SPWM dois níveis (Scilab).

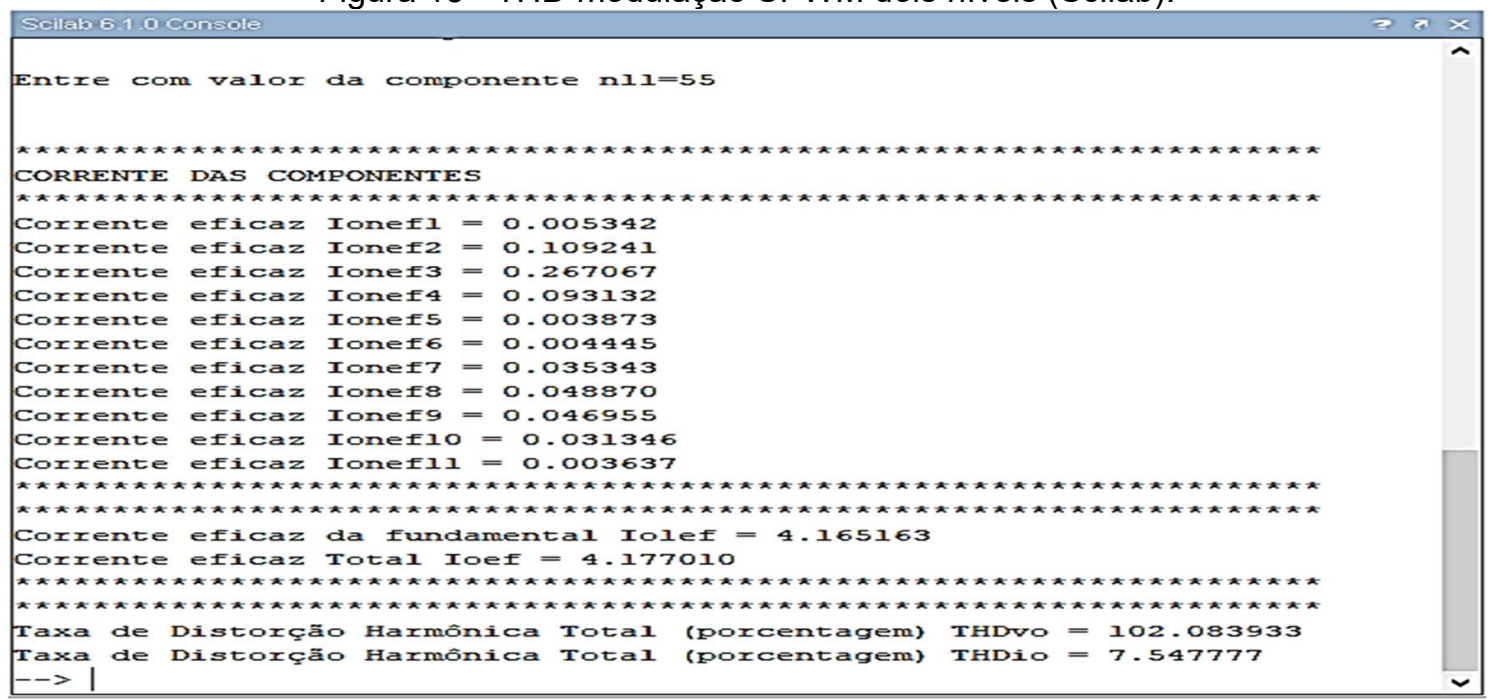

Fonte: Autores 


\subsection{Modulação PWM Senoidal a Três Níveis}

Figura 14 - FFT modulação SPWM três níveis (LTSpice)

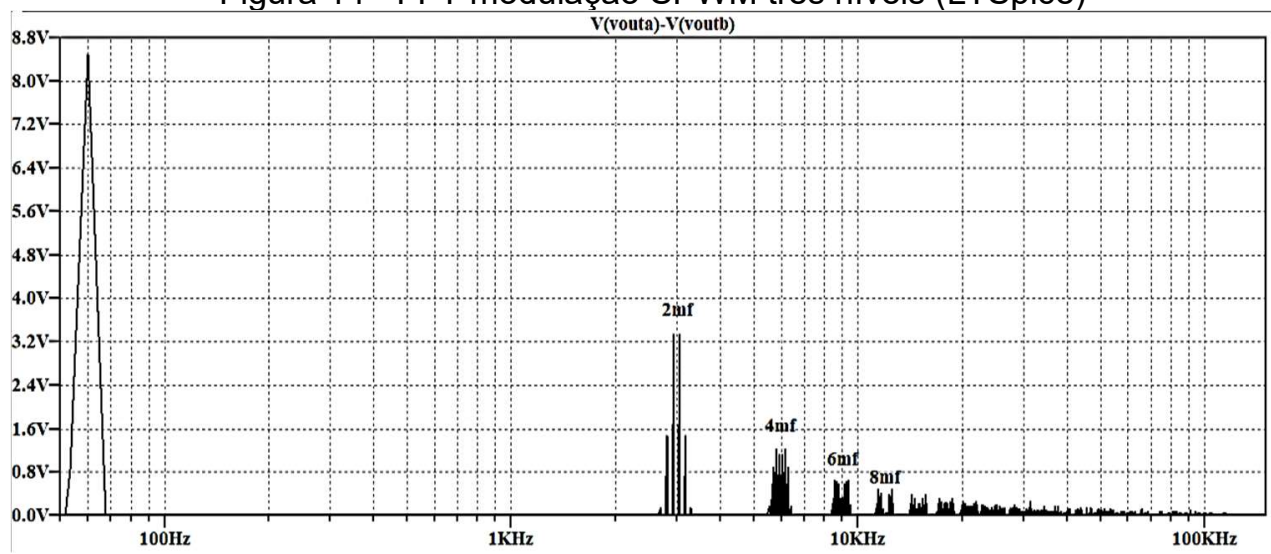

Fonte: Autores.

O resultado do espectro de frequência desta modulação senoidal a três níveis com os valores eficazes das tensões nas regiões de $2 \mathrm{mf}$ e $4 \mathrm{mf}$, seguem abaixo.

Tabela 3 - Valores medidos das componentes (Tensão Eficaz e Vpico).

\begin{tabular}{|c|c|c|c|}
\hline Componentes & $\mathbf{V}\left(\right.$ outA,outB) $\mathbf{n}_{\text {ef }}$ & $\mathbf{V}$ (outA,outB) $\mathbf{n}_{\max }$ & Frequência \\
\hline Fundamental & 8,48 & 12 & 60 \\
\hline $45^{\mathrm{a}}$ & $135,13 \times 10^{-3}$ & $191,10 \times 10^{-3}$ & 2700 \\
\hline $47^{\mathrm{a}}$ & 1,48 & 2,093 & 2820 \\
\hline $49^{\mathrm{a}}$ & 3,33 & 7,7 & 2940 \\
\hline $51^{\mathrm{a}}$ & 3,33 & 7,7 & 3060 \\
\hline $53^{\mathrm{a}}$ & 1,48 & 2,093 & 3180 \\
\hline $55^{\mathrm{a}}$ & $134,40 \times 10^{-3}$ & $190,07 \times 10^{-3}$ & 3300 \\
\hline $93^{\mathrm{a}}$ & $186,47 \times 10^{-3}$ & $263,7 \times 10^{-3}$ & 5580 \\
\hline $95^{\mathrm{a}}$ & $891,36 \times 10^{-3}$ & 1,26 & 5700 \\
\hline $97^{\mathrm{a}}$ & 1,21 & 1,71 & 5820 \\
\hline $99^{\mathrm{a}}$ & 1,12 & 1,58 & 5940 \\
\hline $101^{\mathrm{a}}$ & 1,11 & 1,57 & 6060 \\
\hline $103^{\mathrm{a}}$ & 1,21 & 1,71 & 6180 \\
\hline $105^{\mathrm{a}}$ & $891,73 \times 10^{-3}$ & 1,26 & 6300 \\
\hline $107^{\mathrm{a}}$ & $184,06 \times 10^{-3}$ & $260,3 \times 10^{-3}$ & 6420 \\
\hline
\end{tabular}

Fonte: Autores.

Com os resultados elencados na Tabela 3, foram inseridos os 15 valores de pico das componentes no programa Scilab para obter sua resposta no domínio tempo e frequência e saber qual a tensão instantânea no domínio tempo. 


\section{(c) COBENCE 2021 le Educação em Engenharia 28 a 30 de SETEMBRO

Figura 15 - Tensão instantânea da saída modulação SPWM três níveis (Scilab).

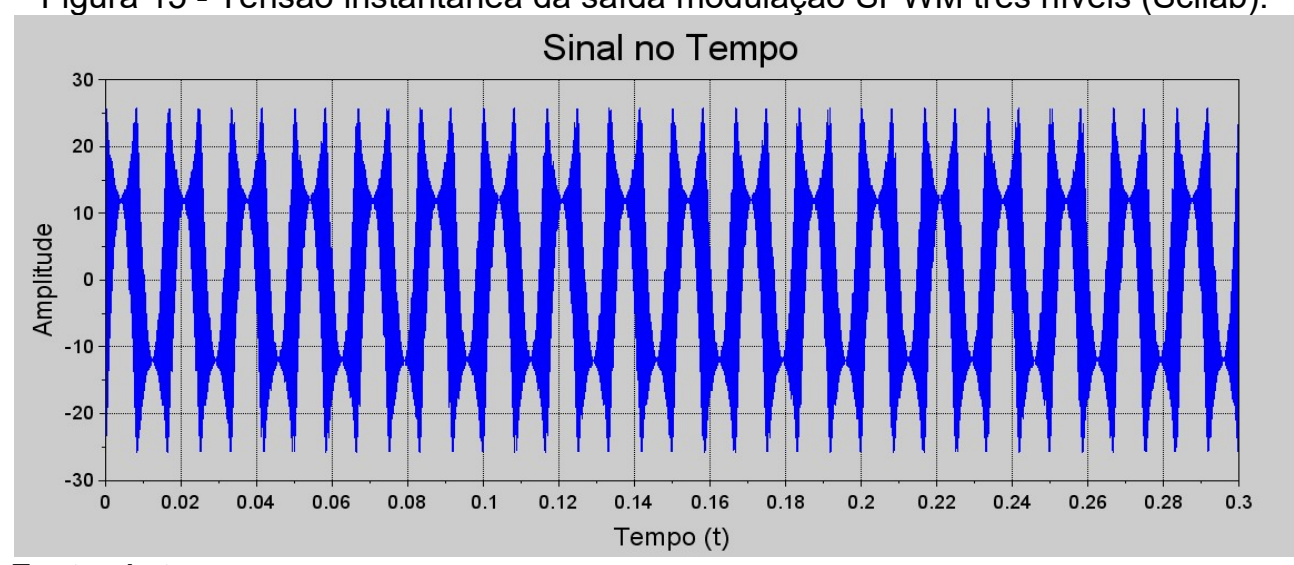

Fonte: Autores.

Analisando o resultado, percebe-se um sinal bastante ruidoso, devido à grande quantidade de sinais de alta frequência sendo somados a fundamental.

Figura 16 - FFT modulação SPWM três níveis (Scilab).

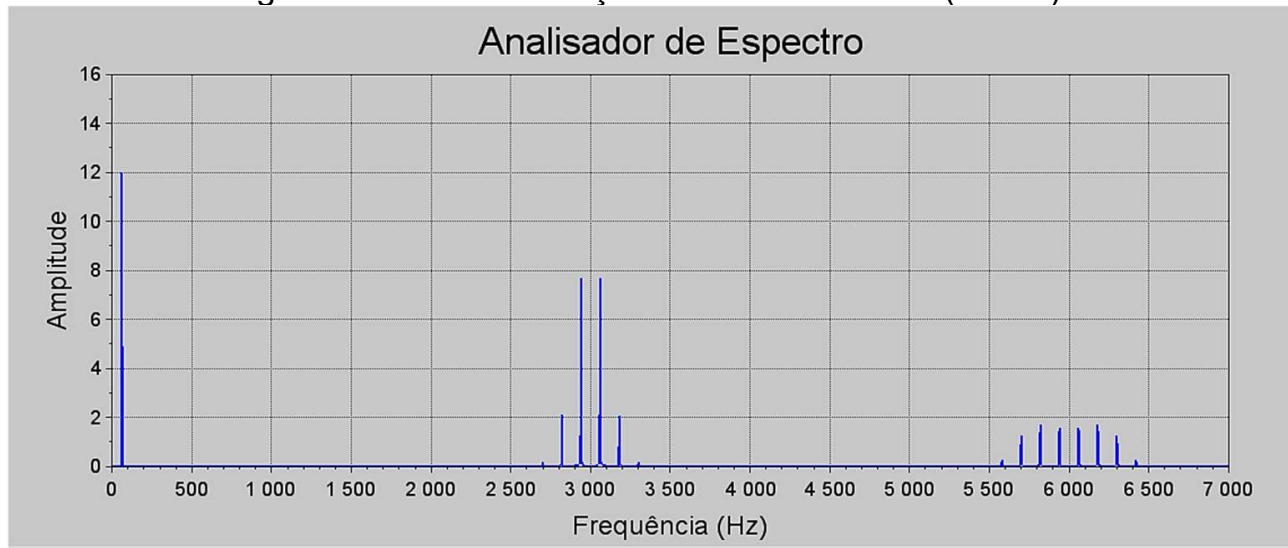

Fonte: Autores.

Figura 17 - Tensão instantânea das 15 componentes individuais (Scilab).

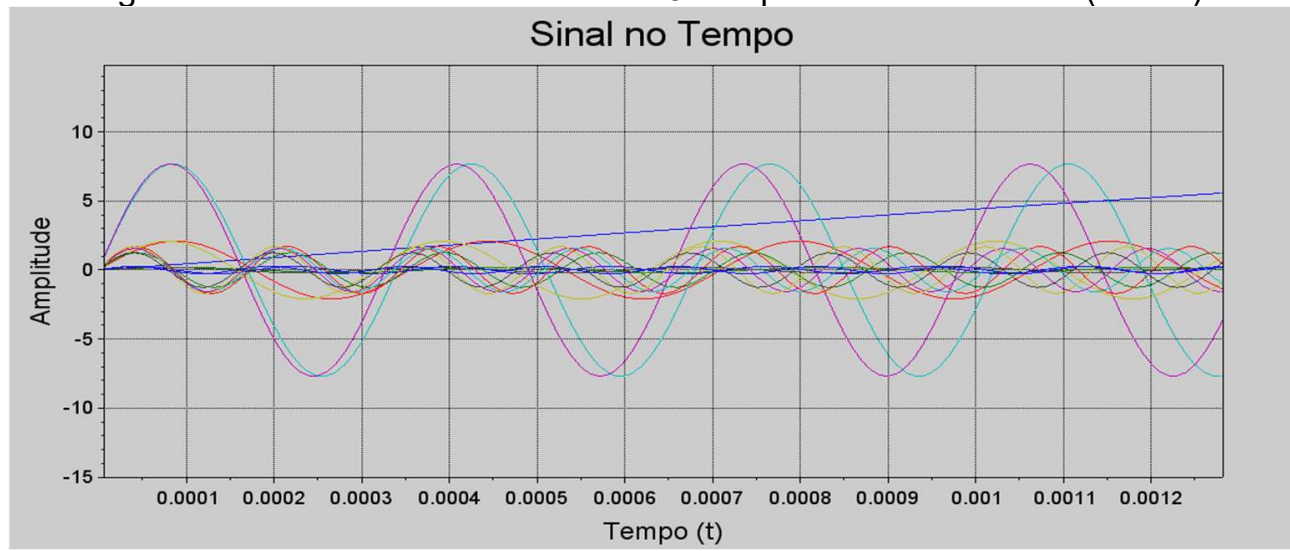

Fonte: Autores.

Inserindo uma carga $\mathrm{R}=2 \mathrm{Ohm}$ e $\mathrm{L}=3 \mathrm{mH}$ no sistema, são verificados os níveis de THDvo e THDio. 
Figura 18 - THD modulação SPWM três níveis (Scilab)

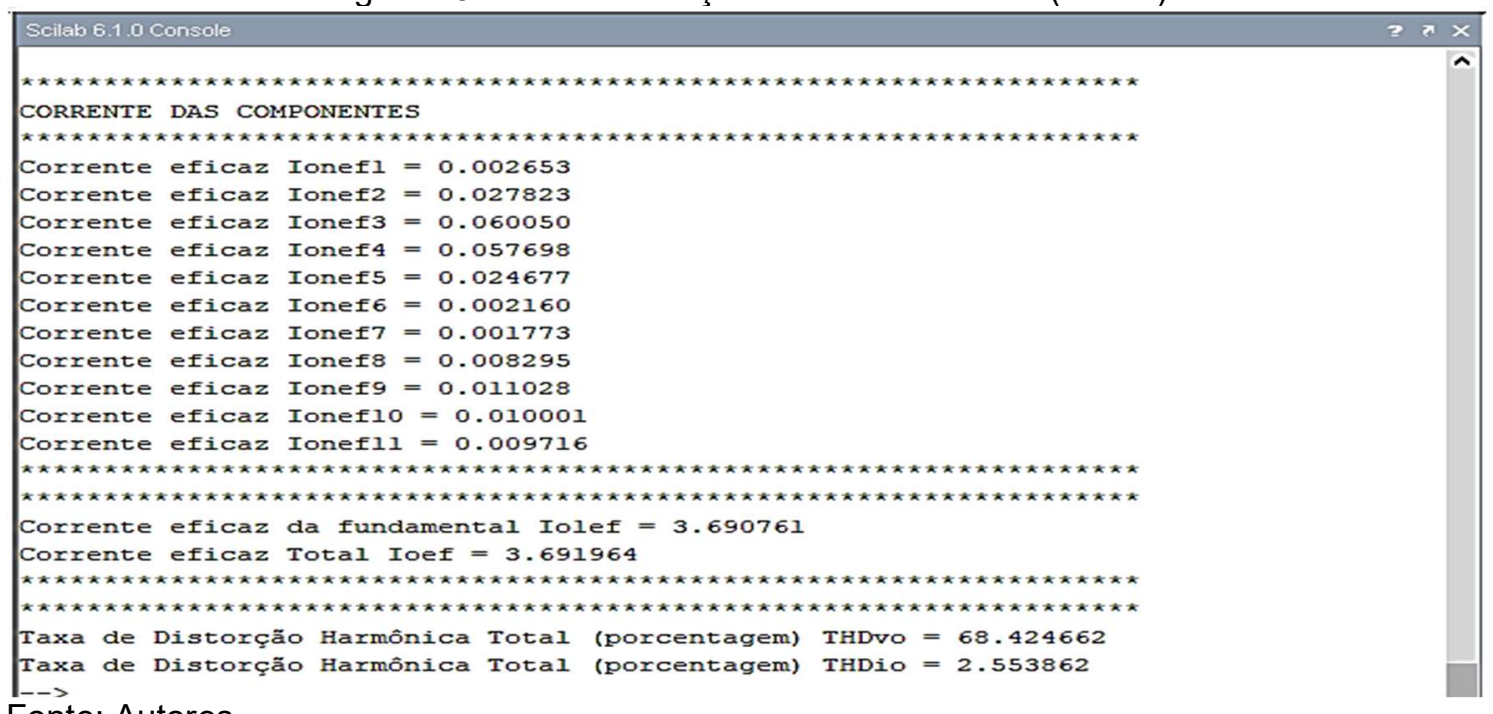

Fonte: Autores.

Fazendo uma breve comparação entre as modulações de dois e três níveis, os resultados demonstraram que na modulação SPWM três níveis, a taxa de distorção de THDvo e THDio caíram aproximadamente pela metade, obtendo uma performance melhor comparado a de dois níveis.

\section{CONSIDERAÇÕES FINAIS}

Esta base teórica aliada às ferramentas gratuitas computacionais LTSPICE e SCILAB, possibilitaram o desenvolvimento deste estudo.

A utilização do software computacional LTSPICE possibilitou a simulação e entendimento de como funciona um sistema que aproveita de forma eficiente o potencial de incidência luminosa em um painel fotovoltaico, além de elaborar circuitos que demonstraram as análises de conversores inversores.

Ao fazer as modelagens com o software SCILAB, considerando todas as variáveis que envolvem o sistema, foi indispensável a transcrição de seus dados físicos em equações matemáticas. Utilizando os modelos das equações foi possível reproduzir o comportamento das componentes.

As Modulações SPWM de Dois e Três Níveis, apesar de apresentarem um THDvo alto em relação à Modulação Controle de Tensão por Defasagem, apresentaram uma aplicabilidade de filtros maior em comparação a outra modulação, uma vez que as componentes harmônicas no sinal, apareceram mais distantes da frequência fundamental. A modulação mais promissora neste sentido foi a Modulação SPWM a três níveis, por ter uma quantidade maior de pulsos por semiciclo em comparação com a dois níveis e tem suas taxas de THDvo e THDio reduzida pela metade.

\section{Agradecimentos}

Ao Professor Mestre Carlos Eduardo e todo corpo docente do Curso Bacharel em Engenharia Elétrica da Universidade Cidade de São Paulo. 


\title{
REFERÊNCIAS
}

D.C. Martins, BARBI Ivo. Eletrônica de Potência: Introdução ao estudo dos conversores CC-CA/ Denizar Cruz Martins, Ivo Barbi. - 3.Ed. ver. - Florianópolis: Ed. Dos Autores, 2011.

FLORERO, H. J., Inversor Monofásico com Modulação PWM Otimizada a Três Níveis, 1989. Dissertação de Mestrado (Mestrado em Engenharia Elétrica) -INEP/EEL, Universidade Federal de Santa Catarina.

LTSPICE: Versão XVII. Software de simulação Spice. Analog Devices, Inc.1995-2020.

RASHID, Muhammad H. Eletrônica de Potência: Circuitos, dispositivos e aplicações / Muhammad H. Rashid; tradução Carlos Alberto Favato; revisão técnica Antonio Pertence Júnior. - São Paulo: Makron Books, 1999.

SCILAB: Versão 6.1.0. Software de código aberto para computação numérica. Grupo ESI, 2017-2020.

\section{Leituras Complementares}

BOYLESTAD, Robert L. NASHELSKY, Louis. Dispositivos eletrônicos e teoria de circuitos. $8^{a}$ ed. São Paulo: Pearson Prentice Hall, 2004.

JOÃO, Tavares Pinho e Marco A GALDINO. Manual de Engenharia para Sistemas Fotovoltaicos. Rio de Janeiro: Cepel-Cresesb, 2014.

PERTENCE, Júnior Antonio Eletrônica analógica: amplificadores operacionais e filtros ativos: teoria, projetos, aplicações e laboratório / Antonio Pertence Júnior. Porto Alegre: Bookman, 2003.

\section{CONTRIBUTION TO THE STUDY OF HARMONICS IN PHOTOVOLTAIC INVERTERS USING SCILAB AND LTSPICE.}

\begin{abstract}
The modeling of electrical systems for power generation encompasses several methods, from the use of computer software to laboratory analysis to ensure the quality of generation. A deeper understanding, through technical and scientific arguments, of on-grid photovoltaic generation systems is of fundamental importance, since this alternative means of energy supply alters the structural pattern of the public and private power grid. In order to measure the impact of these changes, it is proposed the use of two free tools, Scilab in its version 6.1 .0 and the electrical circuits software LTSpice in its version XVII, where it is possible to simulate types of modulations existing in DC-AC voltage inverters, harmonics generated according to each type of modulation and calculations regarding the circuits presented. Such parameters are of fundamental importance in terms of power quality.
\end{abstract}

Keywords: Harmonics. Inverters. Photovoltaics. Modulations. PWM. 